\title{
La ciencia y tecnología y sus indicadores de gestión
}

\author{
Iván Herdoíza*
}

\section{Introducción}

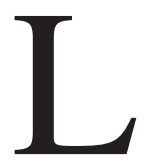

a Ciencia tal como lo afirma la UNESCO, es sólo uno de los muchos sistemas de conocimiento que existen en el planeta. No todo el conocimiento disponible en el mundo pasó necesariamente por la ciencia. En todas las sociedades se ha desarrollado una variedad de enfoques y percepciones para comprender y actuar sobre el entorno, denominados conocimientos ecológicos tradicionales o indígenas o locales y que han constituido un cúmulo de conocimiento ancestral. Todos ellos han contribuido, de una forma u otra, a enriquecer las ciencias modernas ${ }^{1}$.

En los actuales momentos nos encontramos en medio de una civilización globalizada que depende, en altísimo grado, de la ciencia y la tecnología y, por ende, de conocimientos especializados y puntuales, los que deberán estar al servicio de los cambios necesarios para el desarrollo de los pueblos. A fin de poder cuantificar estos cambios requerimos cada vez más de indicadores que nos permitan abalizar el grado de conocimiento y de desarrollo científico que disponemos.

\footnotetext{
* Evaluador económico de proyectos de la Fundación para la ciencia y la tecnología (FUNDACYT) Ecuador.

${ }^{1}$ Sostenibilidad planetaria en la era de la sociedad de la información y del conocimiento. Por un mundo y un futuro sostenible. Camino al 2015. UNESCO. Septiembre del 2003.
} 


\section{Antecedentes}

Como parte del Subprograma XVI del Programa CYTED (Ciencia y Tecnología para el Desarrollo) se creó en 1995 la Red Iberoamericana de Indicadores de Ciencia y Tecnología (RICYT). Esta Red se encarga de la producción y desarrollo de indicadores de ciencia y tecnología para Iberoamérica con el apoyo de los organismos nacionales de ciencia y tecnología de los países que la integran. Esta Red es reconocida por la UNESCO, la OEI y el CAB.

Algunos de los indicadores que se manejan en el ámbito de la ciencia y tecnología y que son auspiciados por la RICYT corresponden a los siguientes: (1) indicadores de actividades científicas y tecnológicas (indicadores de insumo), (2) indicadores de innovación tecnológica, (3) indicadores de cultura científica e impacto social, (4) indicadores de recursos humanos en ciencia y tecnología y de migración de científicos, (5) indicadores bibliométricos, (6) indicadores de la sociedad de la información e (7) indicadores de género.

\section{Problemas institucionales y estándares en la genera- ción de indicadores}

El relevamiento de indicadores de ciencia y tecnología en la región especialmente latinoamericana se ve obstaculizado por problemas de acceso a fuentes de información y validación de los datos. En este contexto el papel de la RICYT consiste en armonizar los conceptos y criterios utilizados en cada país, estandarizando los métodos de trabajo y solucionando problemas a partir de las experiencias de países que ya han arrojado resultados en este campo. Los costos o inversiones en ciencia son tangibles se miden en términos de gasto y recurso humano disponible mientras que los 
resultados o beneficios de la ciencia son más complejos de cuantificar en términos económicos ${ }^{2}$

A nivel internacional varias instituciones se ocupan de este tema, así tenemos en Europa la OCDE (Organización para la Cooperación y el Desarrollo Económico, conformada por 25 países) y EUROSTAT (Oficina de Estadística de las Comunidades Europeas, conformada por 15 países). Prácticamente el "Manual de Frascati" es la única guía internacional que existe sobre la normalización para la toma de datos estadísticos y la medida de inversiones en I+D.

\section{Instituciones encargadas de las estadísticas naciona- les}

En los países iberoamericanos la información se recopila mediante encuestas diseñadas en los organismos nacionales de ciencia y tecnología y en las oficinas de estadísticas nacionales y de acuerdo con las normas y metodología internacional propuestas por la OCDE. Las encuestas se envían a las instituciones públicas, empresas privadas, universidades y ONG que realizan investigación y desarrollo para ser cumplimentadas, analizadas y evaluadas.

La evaluación de los resultados se basa en comparaciones internacionales. Sin embargo, las comparaciones se dificultan por problemas en cuanto a la información y la diferencia en la organización de los sistemas de I+D de cada país. En este sentido la RICYT aporta con sus conocimientos, experiencias y asesoramiento para la aplicabilidad de los estándares internacionales.

\footnotetext{
${ }^{2}$ Indicadores de ciencia y tecnología en Iberoamérica. Agenda 2002.
} 


\section{Indicadores de ciencia y tecnología}

5.1. Encuesta de actividades científicas y tecnológicas $(A C T)$ - Ecuador

En el caso ecuatoriano se ha relevado en dos ocasiones indicadores de I+D: a finales de 1999 se relevaron los primeros indicadores para el período 1996-1998 y a finales del 2004 se actualizaron los indicadores para el período 2001-2003. A continuación se explica la metodología aplicada y se presenta algunos de los resultados obtenidos en la última actualización.

\subsection{Objetivos}

Uno de los objetivos del relevamiento de este tipo de indicadores fue el diseño y construcción de un sistema nacional de indicadores de ciencia, tecnología e innovación que sirva de instrumento de política para la toma de decisiones y cuyos resultados permitan una comparabilidad regional. La encuesta estuvo dirigida especialmente a las universidades, empresas públicas, privadas y organismos no gubernamentales. La encuesta estuvo basada en los fundamentos teóricos y metodológicos del Manual de Frascati y en los criterios e indicadores establecidos por la Red Iberoamericana de Indicadores de Ciencia y Tecnología (RICYT) $)^{3}$. Las actividades básicas que se realizaron durante la encuesta y una vez que se lograron los resultados fueron las siguientes:

- Determinación de instituciones y empresas a ser encuestadas.

${ }^{3}$ Encuesta Nacional de Actividades Científico-Tecnológicas. FUNDACYT 2004. 
- Preparación de los formularios de encuesta

- Establecimiento de las variables a ser medidas

- Aplicación de la encuesta

- Relevamiento de la información

- Codificación de la información

- Estructuración de la base de datos

- Ingreso de datos

- Depuración y validación de la información

- Construcción de indicadores

- Análisis y discusión de resultados finales

- Publicación de indicadores.

Los conceptos que se aplicaron para el diseño de este tipo de encuestas correspondieron a los siguientes:

\subsection{Actividades científicas y tecnológicas (ACT)}

"Actividades sistemáticas estrechamente relacionadas con la producción, promoción, difusión y aplicación de los conocimientos científicos y técnicos en todos los campos de la ciencia y la tecnología. Incluyen actividades tales como la investigación científica y el desarrollo experimental $(I+D)$, la enseñanza y la formación científica y técnica (STET) y los servicios científicos y técnicos (SCT) ${ }^{4}$.

\subsection{Investigación y desarrollo (I+D)}

El Concepto de investigación y desarrollo incluye tres actividades: investigación básica, investigación aplicada y desarrollo experimental.

${ }^{4}$ Medición de las Actividades Científicas y Tecnológicas. Manual de Frascati. Organización para la Cooperación y el Desarrollo Económico. 1993. 


\subsubsection{Investigación básica}

Trabajos experimentales o teóricos que se emprenden para obtener nuevos conocimientos acerca de fenómenos y hechos observables sin pensar en darles alguna aplicación o utilización determinada.

\subsubsection{Investigación aplicada}

Trabajos originales realizados para adquirir nuevos conocimientos. Esta dirigido hacia un objetivo práctico específico.

\subsubsection{Desarrollo experimental}

Trabajos sistemáticos basados en los conocimientos existentes, derivados de la investigación y/o la experiencia práctica, dirigidos a la producción de nuevos materiales, productos o dispositivos, establecimiento de nuevos procesos, sistemas y servicios o mejora sustancial de los existentes.

A continuación se presenta el número de encuestas realizadas y el nivel de respuesta obtenida. 


\section{ENCUESTAS REALIZADAS}

\begin{tabular}{|l|c|c|c|}
\hline \multicolumn{1}{|c|}{$\begin{array}{c}\text { Entidad } \\
(\%)\end{array}$} & Encuestas & $\begin{array}{c}\text { Encuestas } \\
\text { entregadas }\end{array}$ & $\begin{array}{l}\text { Nivel de respuesta } \\
\text { respondidas }\end{array}$ \\
\hline $\begin{array}{l}\text { Universidades } \\
\text { y Politécnicas }\end{array}$ & 59 & 43 & 72,88 \\
\hline $\begin{array}{l}\text { Empresas } \\
\text { públicas }\end{array}$ & 18 & 14 & 77,77 \\
\hline $\begin{array}{l}\text { Empresas } \\
\text { privadas }\end{array}$ & 1275 & 8 & 53,33 \\
\hline $\begin{array}{l}\text { Entidades sin } \\
\text { fines de lucro }\end{array}$ & 119 & 80 & 55,55 \\
\hline Total & 15 & 67,22 \\
\hline
\end{tabular}

\subsection{Resultados arrojados-Ecuador}

Los resultados arrojados por la encuesta de actividades científicas y tecnológicas para el período de estudio corresponden a los siguientes:

\section{Tabla No 1 \\ GASTO EN I+D CON RELACIÓN AL PIB}

Este indicador es el más utilizado para medir la intensidad con que un país apoya a la I+D. En la medida que el resultado de este indicador es más elevado, más importancia le brinda el país a la ciencia y tecnología.

\begin{tabular}{|c|c|c|c|}
\hline & 2001 & 2002 & 2003 \\
\hline Gasto (miles USD) & Gato/PIB (\%) & $15.841,00$ & $18.621,00$ \\
\hline PIB (miles USD) & 21.024 .085 & 24.310 .944 & 27.200 .959 \\
\hline Gasto/PIB (\%) & 0.06 & 0.07 & 0.07 \\
\hline
\end{tabular}


Interpretación: en el caso del Ecuador por cada 100 dólares que se genera como resultado de la actividad económica, el país invirtió en el 2003, \$ 0,07 (siete centavos) para investigación y desarrollo o por cada US\$1.000 el país destinó US\$ 0,70 (setenta centavos). El promedio latinoamericano en el 2001 fue de $0,62 \%$ del PIB. De acuerdo con recomendaciones de la UNESCO debería destinarse por lo menos el $1 \%$ del PIB para actividades de $1+D^{5}$.

\section{Tabla $\mathbf{N}^{\circ} 2$}

\section{PARTICIPACIÓN PORCENTUAL DEL GASTO EN I+D RESPECTO DEL GASTO EN ACT}

Este indicador mide la participación del gasto en I+D respecto del gasto total de todas las actividades científicas y tecnológicas (ACT). Mientras más elevado es este indicador más importancia le brinda el país a la I+D.

\begin{tabular}{|c|c|c|c|c|c|c|}
\hline & $\mathbf{2 0 0 1}$ & $\begin{array}{c}\text { Partici- } \\
\text { pación \% }\end{array}$ & $\mathbf{2 0 0 2}$ & $\begin{array}{c}\text { Partici- } \\
\text { pación \% }\end{array}$ & $\mathbf{2 0 0 3}$ & $\begin{array}{c}\text { Partici- } \\
\text { pación\% }\end{array}$ \\
\hline $\begin{array}{c}\text { Gasto } \\
\text { ACT } \\
\text { (mile USD) }\end{array}$ & 8.618 & 40,65 & 6.474 & 29,01 & 6.156 & 24,85 \\
\hline & 12.583 & 59,35 & 15.841 & 70,99 & 18.621 & 75,15 \\
\hline Total & 21.201 & 100,00 & 22.315 & 100,00 & 24.777 & 100,00 \\
\hline
\end{tabular}

Interpretación: la participación porcentual de I+D en el total de ACT en el año 2001 correspondió al $59.35 \%$. Sin embargo, la tendencia en los siguientes años es a incrementarse la participación de I+D. La media latinoamericana en el período 2001 fue del 75,3\%.

${ }^{5}$ El Estado de la Ciencia. Principales Indicadores de Ciencia y Tecnología Iberoamericanos/Interamericanos. 2002.

164 


\section{Tabla No 3 \\ GASTO EN I+D POR HABITANTE}

Este indicador representa la distribución del gasto total en I+D por habitante. Mientras más elevado el índice mayor participación por habitante.

\begin{tabular}{|l|l|l|l|}
\hline & $\mathbf{2 0 0 1}$ & $\mathbf{2 0 0 2}$ & $\mathbf{2 0 0 3}$ \\
\hline Gasto (miles USD) & 12.583 & 15.841 & 18.621 \\
\hline $\mathbf{N}^{\circ}$ habitantes (miles) & 12.159 & 12.409 & 12.663 \\
\hline Gasto/habitantes (USD) & 1,035 & 1,277 & 1,471 \\
\hline Gasto x (1000 hab.) & $1.034,87$ & $1.276,57$ & $1.470,50$ \\
\hline
\end{tabular}

Interpretación: en el 2003, el gasto en I+D por habitante fue de USD 1,47 o por cada 1.000 habitantes correspondió un gasto de USD 1.471. El promedio latinoamericano se ubicó en el 2001 en USD 2,46 por habitante.

\section{Tabla $N^{\circ} 4$ \\ GASTO EN I+D POR INVESTIGADOR}

Este indicador demuestra la distribución del gasto total por investigador. A su vez demuestra la importancia que brinda el país a la ciencia y tecnología en relación al número de investigadores existentes. Mientras más elevado el indicador mayor participación por investigador.

\begin{tabular}{|l|c|c|c|}
\hline & $\mathbf{2 0 0 1}$ & $\mathbf{2 0 0 2}$ & $\mathbf{2 0 0 3}$ \\
\hline Gasto (miles USD) & 12.583 & 15.841 & 18.621 \\
\hline $\mathbf{N}^{\circ}$ investigadores & 648 & 696 & 845 \\
\hline Gasto/investigadores & 19,42 & 22,76 & 22,04 \\
\hline
\end{tabular}

Interpretación: a cada investigador en el 2003 le correspondió USD 22,04. El promedio latinoamericano en el 2001 fue de USD 78,55. 


\section{Tabla N ${ }^{\circ} 5$}

\section{PARTICIPACIÓN PORCENTUAL DEL GASTO EN I+D POR TIPO DE INVESTIGACIÓN}

Este indicador mide la tendencia y el esfuerzo de un país en la utilización y distribución de los recursos para C\&T en los diferentes tipos de investigación.

\begin{tabular}{|c|c|c|c|c|cc|}
\hline & \multicolumn{2}{|c|}{2001} & \multicolumn{2}{c|}{2002} & \multicolumn{2}{c|}{2003} \\
\hline $\begin{array}{c}\text { Investigación } \\
\text { básica }\end{array}$ & 2.466 & 19,60 & 3.453 & 21.80 & 4.395 & 23,60 \\
\hline $\begin{array}{c}\text { Investigación } \\
\text { aplicada }\end{array}$ & 7.190 & 57,14 & 8.766 & 55,34 & 9.944 & 53,40 \\
\hline $\begin{array}{c}\text { Desarrollo } \\
\text { experimental }\end{array}$ & 2.919 & 23,20 & 3.612 & 22,80 & 4.283 & 23,00 \\
\hline Total & 12.583 & $100,00 \%$ & 15.841 & $100,00 \%$ & 18.621 & $100,00 \%$ \\
\hline
\end{tabular}

Interpretación: en los tres años el tipo de investigación que mayor aporte recibió corresponde a la investigación aplicada que en el 2003 alcanzó el $53,40 \%$. La participación del gasto en desarrollo experimental se mantiene constante y la investigación básica tiende a incrementarse. Una tendencia de distribución mayor o menor hacia la investigación aplicada podría significar que el país esta poniendo mayor o menor esfuerzo hacia la investigación o solución de problemas muy concretos o específicos. La inversión en desarrollo experimental podría significar una tendencia hacia la aplicabilidad de la investigación dentro de un ambiente de innovación tecnológica y vinculada con el sector empresarial. 


\section{Tabla $\mathbf{N}^{\circ} 6$}

\section{PERSONAL EN I+D POR TITULACIÓN \\ DE INVESTIGADORES \\ (Porcentaje de participación)}

Este indicador muestra el personal dedicado a I+D y su distribución por titulo profesional. Una participación importante a nivel de PhD podría indicar que el país esta brindando mayor importancia a la investigación a nivel de país.

\begin{tabular}{|c|c|c|c|c|cc|}
\hline & \multicolumn{2}{|c|}{2001} & \multicolumn{2}{c|}{2002} & \multicolumn{2}{c|}{2003} \\
\hline Profesional & 446 & 68,83 & 451 & 64,80 & 548 & 64,85 \\
\hline Maestría & 141 & 21,76 & 167 & 23,99 & 208 & 24,62 \\
\hline PhD & 60 & 9,26 & 77 & 11,06 & 88 & 10,41 \\
\hline $\begin{array}{c}\text { Total } \\
\text { personal }\end{array}$ & 648 & $100 \%$ & 696 & $100 \%$ & 845 & $100 \%$ \\
\hline
\end{tabular}

Nota: todas las áreas del conocimiento.

Interpretación: del total de investigadores que participaron en el 2003 en actividades de investigación y desarrollo, 10,41\% corresponden a un nivel de PhD. En el 2003 tiende a bajar la participación respecto del 2002, posiblemente por falta de apoyo a programas de capacitación. De los estudios realizados por SENACYT/FUNDACYT la participación de doctorados en investigación está en niveles del $5 \%$. 


\section{Tabla $\mathbf{N}^{\circ} 7$}

\section{INVESTIGADORES POR CADA MIL INTEGRANTES DE LA PEA}

Este indicador muestra el número de personas que se dedican a la investigación y su distribución por cada 1000 habitantes de la Población Económicamente Activa, (PEA). Mientras más elevado el índice, demostraría mayor participación de la C\&T en el desarrollo del país.

\begin{tabular}{|c|c|c|c|}
\hline & $\mathbf{2 0 0 1}$ & $\mathbf{2 0 0 2}$ & $\mathbf{2 0 0 3}$ \\
\hline PEA & $5^{\prime} 173.000$ & $5^{\prime} 329.000$ & $5^{\prime} 400.000$ \\
INVESTIGADORES & 648 & 696 & 845 \\
\hline Investigadores/PEA & 0,125 & 0,1306 & 0,1565 \\
\hline
\end{tabular}

Interpretación: En el 2003 por cada 1000 habitantes hubo 0.16 investigadores. El promedio latinoamericano se ubica en 0.66 investigadores. En el caso ecuatoriano este indicador demuestra poca participación de profesionales investigadores con relación a la PEA, es decir, la población en edad de trabajar.

\subsection{Comparación entre países}

Dentro del marco de negociaciones de un Tratado de Libre Comercio, TLC, con Estados Unidos de Norteamérica es interesante e importante observar el nivel de importancia que brinda cada país involucrado al desarrollo de la ciencia y tecnología.

Tabla $\mathbf{N}^{\circ} 1$

GASTO EN CIENCIA Y TECNOLOGÍA/PIB (Porcentaje)

\begin{tabular}{|l|ll|l|l|l|}
\hline & 1997 & 1998 & 1999 & 2000 & 2001 \\
\hline Ecuador & 0.08 & 0.08 & & & 0.06 \\
\hline Colombia & 0.30 & 0.21 & 0.22 & 0.18 & 0.16 \\
\hline Perú & 0.08 & 0.10 & 0.10 & 0.11 & 0.11 \\
\hline Estados Unidos & 2.55 & 2.58 & 2.63 & 2.68 & 2.76 \\
\hline
\end{tabular}

168 
La participación de la ciencia y tecnología en el PIB de Estados Unidos de Norte América es sensiblemente superior al resto de países de la región. Como referencia el promedio latinoamericano en el 2001 se ubicó en 0,61\%. El gasto en I+D de la región del NAFTA representó en el 2001 el 2,5\%. La Unión Europea destinó el 1,9\% y los países miembros de la APEC destinaron el $1,7 \%$ respecto del PIB.

Tabla No 2

GASTO EN CIENCIA Y TECNOLOGÍA /HABITANTE (Dólares)

\begin{tabular}{|l|r|r|r|r|r|}
\hline & 1997 & 1998 & 1999 & $\mathbf{2 0 0 0}$ & $\mathbf{2 0 0 1}$ \\
\hline Ecuador & 1.33 & 1.23 & & & 1,35 \\
\hline Colombia & 7,29 & 5,14 & 4,13 & 3,63 & 3,14 \\
\hline Perú & 1,97 & 2,23 & 1,94 & 2,25 & 2,19 \\
\hline Estados Unidos & 793,10 & 839,50 & 895,31 & 937,96 & 989,36 \\
\hline
\end{tabular}

Se observa con claridad la diferencia existente del gasto por habitante de los países latinoamericanos frente a los Estados Unidos de Norteamérica.

\section{Tabla $\mathbf{N}^{\circ} 3$ \\ GASTO EN I+D / INVESTIGADOR}

(Dólares)

\begin{tabular}{|l|r|r|r|r|r|}
\hline & 1997 & $\mathbf{1 9 9 8}$ & $\mathbf{1 9 9 9}$ & $\mathbf{2 0 0 0}$ & $\mathbf{2 0 0 1}$ \\
\hline Ecuador & 17,00 & 14,79 & & & 19,42 \\
\hline Colombia & 82,63 & 55,01 & 42,26 & 36,26 & \\
\hline Perú & & & & & \\
\hline Estados Unidos & 190,63 & & 210,48 & & 223,41 \\
\hline
\end{tabular}

Se observa con claridad la diferencia existente del gasto por investigador de los países latinoamericanos frente a los Estados Unidos de Norteamérica. 


\subsection{Indicadores de competitividad}

Frente a una etapa de globalización y negociaciones dentro de un marco de un TLC vale la pena observar como se comporta uno de nuestros principales productos y de mayor exportación en el mundo como es el caso del banano.

Debido a la trayectoria agrícola del país y en un ambiente de competitividad, este sector debería ser el más beneficiado en cuanto a apoyo a la ciencia y tecnología. Sin embargo, observamos que en el caso del banano no es así.

\section{COMPETITIVIDAD DEL BANANO ${ }^{6}$}

(Rendimientos $\mathrm{Hg} / \mathrm{Ha}$ )

\begin{tabular}{|l|r|r|r|r|r|r|}
\hline PAISES & 1995 & 1996 & 1997 & 1998 & 1999 & 2000 \\
\hline Colombia & 295.970 & 291.929 & 308.954 & 307.136 & 311.508 & 311.508 \\
\hline Costa Rica & 440.909 & 461.538 & 467.565 & 532.277 & 540.000 & 540.000 \\
\hline Ecuador & 237.081 & 253.472 & 354.790 & 220.530 & 330.165 & 320.000 \\
\hline Guatemala & 335.714 & 340.500 & 347.619 & 352.000 & 293.018 & 293.018 \\
\hline Honduras & 387.109 & 452.847 & 423.280 & 384544 & 385.549 & 166.954 \\
\hline México & 276.262 & 322.982 & 252.866 & 229.653 & 243.721 & 240.888 \\
\hline Nicaragua & 370.591 & 555.347 & 414.438 & 451.758 & 439.537 & 547.735 \\
\hline Panamá & 454.838 & 441.193 & 421.053 & 361.111 & 394.737 & 624.340 \\
\hline
\end{tabular}

Fuente: apuntes de economía $N^{\circ} 17$. Banco Central del Ecuador.

Interpretación: A pesar de tener elevados costos de producción, entre 1995 y el 2000, Costa Rica es el país con más regularidad en su rendimiento por hectárea y que a su vez le permite obtener mayor competitividad. Ecuador por su gran volumen de producción y explotación extensiva antes que intensiva mantiene menores niveles de productividad que Costa Rica, Nicaragua y Panamá.

\footnotetext{
${ }^{6}$ Apuntes de Economía N ${ }^{\circ}$ 17. Banco Central del Ecuador.
} 


\subsection{Otros indicadores}

Si bien el índice global de competitividad para el crecimiento depende también de otros subíndices, de manera general se colige, y los estudios así lo demuestran, que una mayor inversión en ciencia, tecnología e innovación conduce a mejorar los niveles de competitividad. Los retornos económicos, en el largo plazo, son muy superiores a las inversiones realizadas en investigación científica.

Uno de los indicadores que se utiliza para el cálculo del índice de competitividad para el crecimiento es el subíndice Tecnológico que incluye el grado de innovación y transferencia tecnológica como clave del crecimiento en el largo plazo $^{7}$. En el siguiente cuadro observamos los Índices de competitividad para el crecimiento 2001-2002 de 5 países (entre 75) y su relación con el subíndice tecnológico y el gasto en ciencia y tecnología (el menor valor mejor ubicación).

Tabla $\mathbf{N}^{\circ} 1$

\begin{tabular}{|l|c|c|c|}
\hline País & ÍCC & SIT & Gasto en CyT \\
\hline Chile & 27 & 31 & $0.57(1)$ \\
\hline Brasil & 44 & 35 & $1.05(2)$ \\
\hline Costa Rica & 47 & 32 & $0.35(3)$ \\
\hline Bolivia & 67 & 67 & $0.28(1)$ \\
\hline Ecuador & 68 & 69 & $0.06(1)$ \\
\hline
\end{tabular}

Fuente: Global Competitiveness

Elaboración: autor

(1) 2001; (2) 2000; (3) 1998

${ }^{7}$ Global Competitiveness Report. Índice de Competitividad para el Crecimiento. 2001-2002. 
Para entender mejor la influencia de la inversión en ciencia y tecnología se presenta el caso de los subíndices (SI) tecnológicos de varios países para el período 20012002 y su relación con otros SI. En general se aprecia que los subíndices tecnológicos tienen una estrecha relación con el resto de subíndices y por lo tanto es determinante para su ubicación en el ranking mundial.

\section{Tabla N $N^{\circ} 2$ SUBÍNDICES}

\begin{tabular}{|l|r|r|r|}
\hline Países & $\begin{array}{c}\text { Contexto } \\
\text { Macroeconómico }\end{array}$ & Institucional & Tecnológico \\
\hline Estados Unidos & 14 & 17 & 1 \\
\hline Finlandia & 2 & 2 & 2 \\
\hline Taiwán & 18 & 21 & 3 \\
\hline Suecia & 8 & 7 & 4 \\
\hline Dinamarca & 5 & 1 & 8 \\
\hline Chile & 35 & 19 & 31 \\
\hline Brasil & 75 & 53 & 35 \\
\hline México & 54 & 50 & 43 \\
\hline Colombia & 66 & 60 & 60 \\
\hline Perú & 58 & 54 & 61 \\
\hline Angola & 101 & 91 & 98 \\
\hline Chad & 96 & 101 & 102 \\
\hline Haití & 99 & 102 & 101 \\
\hline
\end{tabular}

Fuente: Global Competitiveness Report. Índice de Competitividad para el Crecimiento. 2001-2002.

Elaboración: autor

A modo de conclusión podríamos decir que en el caso del Ecuador el gasto en ciencia y tecnología es muy reducido a pesar de ser un tema trascendente y estrechamente vinculado con la competitividad y más aún dentro del contexto de un Tratado de Libre Comercio con los Estados Unidos de Norteamérica. La ciencia y tecnología no constituyen una política de Estado. 


\section{La innovación tecnológica}

En el actual escenario de globalización el recurso humano, la tecnología y la competitividad constituyen la parte esencial de cualquier modelo de desarrollo. La innovación tecnológica es un proceso concebido inicialmente como un modelo lineal de flujo de conocimiento, donde la innovación es la consecuencia del desarrollo de nuevas ideas que se transforman en productos a través de la investigación científica básica, pasando por el desarrollo experimental, hasta la fabricación y comercialización del nuevo producto. Según este modelo la I+D es principal indicador de la actividad de innovación en su conjunto.

Este concepto lineal de innovación tecnológica ha cambiado radicalmente en los últimos años, siendo sustituido por un modelo de conexión en cadena más complejo, según el cual las actividades de innovación son el resultado de un complejo proceso de retroalimentación de fuertes interacciones continuas y repetidas entre diferentes elementos heterogéneos e interdependientes tales como: investigación y desarrollo, ingeniería, estudios de mercado y de usuarios, planificación financiera, canales de distribución, proveedores de equipos y materias primas, ventas, etc.

El tener información sobre la evolución de los procesos de innovación se ha hecho imprescindible para la política tecnológica. Sin embargo, no es una tarea fácil. El término innovación es un término ambiguo considera a un proceso como a un resultado, este concepto tiene dimensiones muy diferentes, se puede considerar innovación tanto un avance radical, como puede ser una nueva vacuna o un nuevo tipo de embalaje o la mejora en la puesta en el mercado de un determinado producto, etc. Para esto es imprescindible la utilización de indicadores de innovación estandarizados que midan con cierta precisión esta compleja actividad. 
El Manual de Oslo publicado por la OCDE proporciona definiciones y la metodología para diseñar encuestas que recojan los datos sobre fuentes de ideas innovadoras, inversiones e impacto de la innovación. En el caso de los países latinoamericanos se ha creado el Manual de Bogotá que brinda normas y definiciones para la medición de la actividad innovadora en Iberoamérica. La innovación tecnológica se mide también en las tareas de la organización interna y de gestión de la empresa, sobre todo de las actividades relacionadas con la informatización y acceso a redes, pero también en aspectos de protección del medio ambiente, higiene, prevención, de riesgos, etc. que pueden suponer importantes beneficios económicos para la empresa y un claro aumento de su competencia.

\subsection{Distinción de las innovaciones}

Pueden distinguirse entre innovaciones progresivas 0 incrementales o innovaciones totales o radicales. Las innovaciones progresivas se refieren a las mejoras sucesivas a las que son sometidos todos los productos o procesos. Este tipo de cambios observa el incremento general de la productividad, mejora la eficiencia técnica y la precisión en los procesos. El ritmo de mejora es lento en un principio, adquiere su madurez y posteriormente enfrenta rendimientos decrecientes hasta que aparezca una innovación radical que permita nuevos niveles de productividad.

La innovación radical consiste en la introducción de un producto o proceso totalmente nuevo. Es imposible que una innovación radical se dé como resultado de las mejoras de una tecnología existente. Por tanto, este concepto se refiere a un producto que presenta diferencias significativas respecto a los fabricados anteriormente en cuanto a su finalidad, prestaciones, características, propiedades teóricas o materias primas y componentes utilizados en su fabricación. 
La innovación de proceso denota la adopción de métodos de producción nuevos o sensiblemente mejorados. Puede resultar de modificaciones en el equipo o en la organización de la fabricación o de estas dos modificaciones asociadas. Los métodos introducidos pueden estar destinados a la producción de productos nuevos o mejorados o a fabricar productos existentes de forma más eficiente. Hasta que una innovación no es introducida en el mercado (innovación de producto) o utilizada en un proceso de producción (innovación de proceso) no debe ser considerada como tal en términos estrictos.

Muchas empresas no desarrollan nuevos productos o procesos sino que centran sus esfuerzos en la asimilación de tecnologías concebidas en economías más desarrolladas. Este proceso de asimilación requiere de adaptaciones al medio local y cambios de organización. En consecuencia, en estos países la medición del cambio tecnológico no puede prescindir de la evaluación de elementos que, en otros contextos, pueden resultar poco significativos o marginales.

Las nuevas teorías del crecimiento endógeno destacan la influencia que tiene sobre las tasas de crecimiento factores tales como la I+D o la creación de conocimiento. EI proceso de innovación no es un hecho aislado, sino que tiene significativas repercusiones sobre otras variables de extrema importancia como el crecimiento, la productividad y el empleo. En este sentido, el análisis de impacto no puede limitarse a la firma o a un nivel macroeconómico, sino que debe incluir la dimensión macro ${ }^{8}$.

\subsection{Experiencias de encuestas de innovación tecnológica en Latinoamérica}

Algunas de las encuestas realizadas en el sector industrial en América Latina corresponden a encuestas

${ }^{8}$ PÉREZ, Carlota. Las Nuevas Tecnologías una Visión de Conjunto. 2005. 
nacionales y cuya cobertura muestral admite comparación de resultados, así tenemos las siguientes:

\section{NÚMERO DE ENCUESTAS POR PAÍS}

\begin{tabular}{|c|l|r|}
\hline Año & País & Número de encuestas \\
\hline 1997 & México & 1.322 \\
\hline 1996 & Colombia & 885 \\
\hline 1997 & Venezuela & 1.382 \\
\hline 1997 & Argentina & 1.639 \\
\hline 1995 & Chile & 541 \\
\hline 1986 & Uruguay & 261 \\
\hline
\end{tabular}

Fuente: La innovación realmente existe en América Latina: medidas y lecturas. Versión preliminar. Judith Sutz. 2000. Elaboración: autor

Sobre la base de los resultados arrojados se puede inferir que la "empresa innovadora" es aquella que hizo esfuerzos para la obtención de productos y/o procesos nuevos o sensiblemente modificados y que los esfuerzos para concretar la innovación se centraron especialmente en actividades de I+D.

El resultado de estas encuestas permite observar que el núcleo de la innovación en las empresas se encuentra dentro de la empresa, las ideas provienen de la misma empresa $y$, por tanto, las fuentes externas son muy poco utilizadas. Esto podría deberse básicamente a tres razones:

1. Lo que existe fuera de la empresa es muy insignificante para fines de la industria.

2. las empresas son demasiado débiles internamente como para sacar alguna ventaja de la oferta de conocimientos de su entorno. 
3. lo que se ofrece en el medio local no es irrelevante y tampoco las empresas son débiles sino lo que es esencialmente débil son los sistemas de innovación, es decir el conjunto de articulaciones que podrían relacionar a las empresas con su entorno, básicamente la relación empresa-universidad ${ }^{9}$.

\subsection{Encuesta de demanda tecnológica en el ecuador}

A finales del 2001 FUNDACYT obtuvo resultados de una encuesta nacional de demanda tecnológica dirigida al sector empresarial en el Ecuador. El trabajo incluyó una encuesta de aproximadamente 600 empresas consideradas las más grandes desde le punto de vista del volumen de ventas. La encuesta no estuvo enmarcada dentro de los estándares latinoamericanos que permitan realizar una comparabilidad entre países. Sin embargo, estuvo dirigida hacia conocer ciertos aspectos cualitativos y cuantitativos que permitan conocer las necesidades básicas de innovación tecnológica y que podrían constituir una información básica para el organismo de ciencia y tecnología del Ecuador en su afán de apoyar a la innovación tecnológica.

\subsubsection{Demanda en innovación tecnológica}

De la encuesta nacional de demanda de innovación tecnológica se obtuvieron los siguientes resultados ${ }^{10}$ :

1. El $26 \%$ de las empresas encuestadas consideran que en las siguientes actividades donde se manejan los procesos de producción de la empresa hay mayor interés para

9 La innovación realmente existe en América Latina: medidas y lecturas. Versión preliminar. Judith Sutz. 2000.

${ }^{10}$ Informe final. Encuesta de demanda de innovación tecnológica. FUNDACYT. Diciembre 2001. 
la implantación de mejoras tecnológicas: mejoramiento de la calidad ( $32 \%$ de las encuestas así lo consideran), incremento de la productividad (28\%) y disminución de los costos de producción (25\%).

Sin embargo, hay otras áreas hacia las cuales dedican un tiempo importante para la innovación como es el caso de la organización y administración (17\%); diseño de productos $(16 \%)$ e información y comunicación $(16 \%)$.

2. El $78 \%$ de las empresas encuestadas consideran que sus productos son competitivos a nivel nacional. El $42 \%$ de las empresas encuestadas consideran que son competitivas en el ámbito internacional. Considerando que en el estudio se incorpora a buena parte de las empresas de tamaño grande sería imperativo que se establezcan mecanismos para mejorar la productividad y calidad de los bienes y servicios producidos a fin de que puedan ser competitivos en el ámbito internacional.

3. Respecto de la maquinaria y equipo que utilizan las empresas, el mayor problema se enmarca en la subutilización de la capacidad instalada $(24 \%$ de los encuestados); poca capacidad de producción (23\%) y la obsolescencia del equipo (18\%).

Probablemente, esto es el resultado de problemas de carácter estructural que se arrastran desde varias décadas atrás a raíz de las políticas dictadas por la CEPAL, donde se pretendió afianzar un modelo económico bajo el esquema de sustitución de importaciones y la importación de tecnología de última generación (para la época que transcurría) y la utilización de la materia prima nacional. Sin embargo, el Estado no apoyó el desarrollo científico y tecnológico y peor aún la innovación tecnológica, Las empresas y su equipamiento se sobre- 
dimensionó, el mercado nacional era pequeño y necesariamente hubo que importar materia prima, accesorios, repuestos y tecnología.

En las actuales circunstancias es probable que muchas empresas mantengan aún tecnología obsoleta como así lo demuestra el $18 \%$ de los encuestados. Sin embargo, existirán otras empresas que se habrán tecnificado en el transcurrir del tiempo pero que debido a las crisis del país, en lo últimos años, se han visto afectadas con un mercado (escasa demanda) que no ha justificado su inversión en tecnología. Más aún, habrá que pensar bajo el paraguas del sistema de la dolarización donde se observa, de manera general, que la industria nacional no es competitiva - en precio y calidad - frente a los mercados internacionales y así como también en el marco de los tratados de libre comercio.

4. El resultado de la encuesta demuestra que el proceso de control de calidad es el más desarrollado en las empresas (43\% de los encuestados); gestión ambiental (23\%) y la reingeniería de procesos (18\%). Podríamos decir que se está creando conciencia respecto de estos tres procesos que probablemente hasta hace poco tiempo atrás no eran tomados en cuenta. Probablemente, entre otros, se debe a las exigencias internacionales en cuanto al precio - calidad de los productos, certificaciones de normas de calidad y mejoramiento continuo reconocidas internacionalmente y mecanismos de uso de tecnologías limpias y control ambiental.

5. Solo el $72 \%$ de las empresas consideran que sus servicios de posventa son aceptables y sólo el $16 \%$ los consideran excelentes. Este resultado permite observar que los servicios de apoyo y respaldo para el cliente no son, 
en la mayoría de los casos, competentes y que faltaría bastante preparación, en este campo, como para competir internacionalmente.

\subsubsection{Demanda en necesidades tecnológicas.}

Según los empresarios, los aspectos más importantes que apoyarían a mejorar la producción y productividad sería: decremento en los precios de las materias primas $(21 \%$ de los encuestados); disminución en el tiempo de procesos productivos $16 \%$; reducción en el desperdicio de materia prima 14\%; precios bajos de la maquinaria $11 \%$; ahorro de energía $11 \%$; mayor rendimiento en insumos y maquinaria $11 \%$ y sustitución de insumos importados por nacionales $6 \%$.

No es relevante, para el empresario, la sustitución de insumos importados por nacionales esto podría deberse a dos situaciones: es muy pequeña la utilización de insumos importados que no amerita preocuparse o hay desconfianza o escasez de los insumos nacionales que se prefiere $u$ obliga a continuar consumiendo los importados. Probablemente es una combinación de ambos.

2. Hay otras áreas que también son de importancia dentro de las empresas como es el caso de la planificación y mantenimiento de inventarios y productos (15\%); mitigación de impactos ambientales (10\%); embalaje y movilización $(9 \%)$ y fabricación y cultivo $(9 \%)$.

Las actividades empresariales más costosas son: planificación y control de la producción $21 \%$; fabricación o cultivo $21 \%$; planificación y mantenimiento de inventarios y productos $18 \%$; embalaje y movilización $17 \%$.

3. El $58 \%$ de las empresas conocen de mejoras tecnológicas con respecto a las que utilizan actualmente, sin 
embargo, el $46 \%$ de estas empresas no han mejorado su tecnología a pesar de poder obtenerla, debido a que su proceso productivo actual no demanda de cambios sustanciales, probablemente su nicho de mercado es el nacional y no requieren de mayor tecnología e innovación. Adicionalmente el $88 \%$ de las empresas bien informadas no pueden acceder a nueva tecnología por falta de recursos económicos. El 37\% no conocen de nuevas tecnologías.

Definitivamente, estas cifras tendrán que transformarse o invertirse en los próximos años. El mismo sistema económico actual y las exigencias de la globalización se encargarán de mantener ese equilibrio, como un tamiz, donde permanecerán las empresas e industrias competitivas (precio y calidad).

4. La encuesta refleja que las empresas adquieren información tecnológica básicamente de revistas especializadas $23 \%$; ferias o exposiciones $23 \%$; Internet $21 \%$; expertos $19 \%$ y seminarios $11 \%$. Básicamente, luego de las revistas y ferias, es el Internet el medio más utilizado para obtener información.

Los empresarios no acceden a las universidades para obtener información (1\%). Apenas el $24 \%$ de las empresas conocen de la existencia de centros de investigación en las universidades y el $72 \%$ manifiestan el desconocimiento de estos centros. Solo el $13 \%$ de empresas han recibido asistencia de centros de investigación nacional. Esto se complementa con el resultado de que el $50 \%$ de las empresas, que responden, han recibido asistencia técnica nacional y el $49 \%$ asistencia técnica extranjera.

Las universidades y centros de investigación nacional son muy poco conocidos y de acuerdo con la experiencia en el primer Programa Nacional de Ciencia y Tec- 
nología los centros de investigación prestan servicios más por razones coyunturales que por obedecer a una planificación de una agenda o cartera de proyectos de estos centros.

6.3.3. Investigación en ciencia y tecnología. Disposición a invertir en ciencia y tecnología

1. Es interesante el conocer que el $27 \%$ de las empresas encuestadas disponen de alguna unidad de investigación y desarrollo, esto daría la idea de la importancia que están dando, un buen número de empresas, a la formación de este tipo de unidades. Sin embargo, el comportamiento general y que corresponde al $71 \%$ de los encuestados no disponen de este tipo de unidades.

2. El $61 \%$ de las empresas invirtieron entre el 0 y $5 \%$ en capacitación (19\% de ellos no invirtieron en este rubro). Definitivamente la capacitación es un rubro de muy poca importancia dentro de las empresas. El 58\% de las empresas consideran que debe incrementarse el porcentaje de inversión en capacitación.

3. El $58 \%$ de los empresarios se muestra dispuesto a financiar inversiones en su empresa. El $26 \%$ en su sector empresarial y el $16 \%$ en su país en general. Esto podría demostrar la mentalidad del empresario por crecer pero individualmente o familiarmente.

4. El principal incentivo para financiar inversiones en innovación tecnológica es la tasa de interés $24 \%$, el contar con personal en investigación y desarrollo $22 \%$, un plazo mayor que el crédito corriente $17 \%$, condonación parcial de la deuda por innovación tecnológica $16 \%$ y contar con centros de desarrollo tecnológico 16\%. 


\section{Conclusiones}

1. El estudio realizado no muestra tendencias históricas respecto de lo sucedido o la situación de la demanda tecnológica de años anteriores. Sin embargo, las tendencias actuales son bastante desalentadoras sobre la importancia que brinda el sector productivo a la investigación y a la innovación tecnológica en el Ecuador.

2. Básicamente se observan áreas generales donde se podría apoyar la investigación, así tenemos las siguientes:

Demanda de Innovación tecnológica en su orden tenemos: (1) mejoramiento y control de la calidad, (2) aumento de la productividad, (3) disminución de los costos de producción, (4) organización y administración, (5) diseño de productos e (6) información y comunicación.

En el proceso productivo se destacan, en su orden; (1) gestión ambiental y (2) reingeniería de procesos. En cuanto a maquinaria y equipo: (1) subutilización de la capacidad instalada, (2) poca capacidad de producción y (3) obsolescencia del equipo.

Necesidades tecnológicas en su orden tenemos: (1) disminución de tiempos en los procesos productivos, (2) reducción del desperdicio de materia prima, (3) ahorro de energía, (4) mayor rendimiento en insumos y maquinaria, (5) sustitución de insumos importados por nacionales, (6) mitigación de impactos ambientales, (7) embalaje y movilización (entre las actividades empresariales más costosas) y (8) fabricación y cultivo (entre las actividades empresariales más costosas).

3. Es urgente que el país tome una decisión respecto del apoyo a la ciencia y tecnología como una política de Estado más aún en un contexto de un Tratado de Libre 
Comercio frente a Estados Unidos de Norteamérica. Los indicadores tanto de actividades de ciencia y tecnología como de innovación tecnológica nos ponen de manifiesto una posición poca alentadora del país frente a un mundo globalizado donde la tecnología, la competitividad y el recurso humano son parte esencial de su desarrollo.

Las últimas declaraciones del actual Gobierno permiten observar cierto interés en apoyar este sector, sin embargo, esta deberá obedecer a una planificación de largo plazo con un organismo que tenga la suficiente capacidad y experiencia para organizar y gestionar las permanentes interacciones de las diferentes entidades que conforman un Sistema Nacional de Ciencia, Tecnología e Innovación. 